\title{
Prolonged follow-up of colorectal cancer patients after 5 years: to follow or not to follow, that is the question (and how)!
}

\author{
Raul Caso ${ }^{1}$, Anne Fabrizio ${ }^{2}$, Michael Sosin ${ }^{3}$ \\ ${ }^{1}$ Department of Surgery, MedStar Georgetown University Hospital, Washington, DC, USA; ${ }^{2}$ Department of Surgery, Colorectal Division, Beth \\ Israel Deaconess Medical Center, Boston, MA, USA; ${ }^{3}$ Hansjörg Wyss Department of Plastic Surgery, NYU Langone Health, New York, NY, USA \\ Correspondence to: Michael Sosin, MD. Hansjörg Wyss Department of Plastic Surgery, NYU Langone Health, New York, NY, USA. \\ Email: sosinmi@gmail.com. \\ Provenance and Peer Review: This article was commissioned by the Editorial Office, Annals of Translational Medicine. The article did not undergo \\ external peer review. \\ Comment on: Kong L, Peng J, Li J, et al. Prolonged surveillance of colorectal cancer patients after curative surgeries beyond five years of follow-up. \\ Ann Transl Med 2019. doi: 10.21037/atm.2019.10.39.
}

Submitted Oct 24, 2019. Accepted for publication Nov 06, 2019.

doi: $10.21037 / \mathrm{atm} .2019 .11 .40$

View this article at: http://dx.doi.org/10.21037/atm.2019.11.40

It is estimated that in 2018 there were 1.8 million patients diagnosed with colorectal cancer (CRC) worldwide resulting in 862,000 deaths, which is an increase from 2012 by $33 \%$ and $24 \%$, respectively (1). Surgical resection remains the standard of care for resectable, non-metastatic, CRC. Following complete resection, more than $90 \%$ of recurrences occur in the first 5 years with most occurring in the first 3 years (2). Beyond 5 years, however, there are currently no clear recommendations. There are some studies with up to 10 years of follow-up after surgery that have reported late recurrences after 5 years (3), thus the utility of prolonged follow-up after the initial 5 years is an important question. To address this knowledge gap, Kong et al. investigated the efficacy of prolonged follow-up beyond 5 years using a retrospective cohort of 1,054 CRC patients who underwent radical resection from 1980 to 1996 at their institution (4). The patient cohort consisted of $15 \%$ rightsided colon tumors, $18 \%$ left-sided colon tumors, and $62 \%$ rectal tumors. Pathological stages included 14\% stage I, 56\% stage II, and $31 \%$ stage III. Patients were followed every 3 months for the first 2 years, every 6 months in the following 3 years, and then yearly. To detect local or distant recurrence, the authors utilized a physical exam, ultrasound, X-ray, and computed tomography. The authors found significant associations between survival and the following variables: age $(\mathrm{P}=0.026)$, tumor site $(\mathrm{P}<0.001)$, and pathologic stage $(\mathrm{P}<0.001)$. Compared to patients who died within 5 years of surgery, patients who survived past 15 years had a higher proportion of colon cancer relative to rectal cancer and stage I/II disease. Furthermore, $91 \%$ of relapses occurred in the first 5 years after surgery, whereas less than $1 \%$ of patients relapsed after 15 years of surveillance.

Since the mid-1980s, the death rate from CRC has declined resulting in a large number of CRC survivors (5). Of the 13.7 million cancer survivors living in the United States as of January 2012, 9\% were CRC survivors (6), therefore determining an optimal surveillance protocol for CRC survivors is necessary due to the significant burden follow-up can pose on patients, physicians, and the healthcare system alike. The objective of follow-up programs for long-term CRC survivors is the early-stage detection of disease recurrence, at the point where it is amenable to curative resection. In their study, Kong et al. confirm much of the data already known-approximately $90 \%$ of CRC recurrences will occur in the first 5 years after resection and recurrence beyond the initial 5 years of follow-up is less common. In their cohort, less than $1 \%$ of recurrences occurred after 15 years. Perhaps the more relevant finding was the $6 \%$ recurrence rate that occurred between 5 and 10 years of follow-up which the authors failed to emphasize. Although a relatively small percentage compared to the $91 \%$ recurrence rate in the first 5 years after resection, a $6 \%$ recurrence rate is not insignificant. It would have been informative for the reader if a comparison had been performed of clinicopathologic characteristics between patients that recurred in the first 5 years versus 
those that recurred after 5 years of resection. This information might have some clinical relevance in that it can help identify patients that are still at risk of recurrence after the initial 5 years of surveillance. After 15 years of surveillance, the authors found a recurrence rate of $0.8 \%$ among their cohort. Given such a low recurrence rate, is it realistic that all CRC patients be followed for such extended periods of time? One important question not addressed by the authors is the burden this would put on healthcare systems, as it relates to costs of extended follow-up.

Currently the standard duration of follow-up after CRC resection is 5 years, on the basis of the American Society of Colon and Rectal Surgeons and American Joint Committee on Cancer, which provides surveillance practice guidelines including physical examination, CEA, CT scan, colonoscopy, and endorectal ultrasound (7). However, establishing an optimal length of follow-up in accordance with risk stratification assigned to each individual patient is ideal. Frontali et al. addressed this specific question in a recent study (8). The authors assessed the utility of prolonged follow-up more than 5 years after CRC resection in a cohort of patients without evidence of recurrence during the initial 5 years of surveillance. After 5 years, Frontali et al. noted a tumor recurrence rate of $7 \%$ with a higher incidence among rectal cancer patients versus colon cancer patients ( $11 \%$ vs. $2 \%, \mathrm{P}=0.014)$. Among CRC patients with recurrences after 5 years, the authors noted high recurrence rates in colon cancer patients with pT3 tumors, in rectal cancer patients with ypT0 to ypT2 tumors, and in rectal cancer patients with ypN0 tumors (8). Given the findings by Frontali et al., it might be reasonable to follow all rectal cancer patients and select colon cancer patients with locally advanced disease for longer than 5 years after curative resection. Some of the data presented by Frontali et al. is corroborated by Kong et al., however the study by Kong et al. lacks some granularity. Kong et al. concluded that surveillance can perhaps end at 15 years of follow-up but fail to provide the reader with specific patient cohorts that might benefit from a more extended followup protocol. Additionally, although clinicopathologic characteristics have been linked to recurrence, incorporating molecular markers for prognostic prediction, as has been applied in a wide array of cancer types (9), might improve our ability to successfully stratify patients after resection. In CRC, microsatellite instability and mutation status in $K R A S, N R A S$, and BRAF have been proposed as potential prognostic factors $(10,11)$. Ultimately, clinicopathologic and genomic data will help guide not only adjuvant therapy assignment but they will tailor surveillance protocols at the individual patient level.

There are a few areas where the study by Kong et al. is lacking some important details. The authors do not provide any information regarding neoadjuvant or adjuvant therapy. This information is important for the reader but also allows proper stratification of patients at risk for recurrence as has been previously reported (12). Additionally, the authors fail to account for the impact total mesorectal excision (TME) may have had on the outcomes of rectal cancer patients in their study. Although TME was first described in 1979, it did not become standard of care until the 2000s. TME has a has been shown to impact local recurrence and is a big advancement in the management of rectal cancer patients, which comprised a majority of the patient cohort in the study by Kong et al. Furthermore, the authors do not provide information regarding routine CEA labs or colonoscopy during followup, which are, in addition to routine computed tomography and physical exams, currently accepted surveillance modalities $(13,14)$. In comparing clinicopathologic characteristics between patients surviving less than 5 years and over 15 years, the authors failed to differentiate between right- and left-sided colon cancers, which are known to have differing prognostic values (15). Additionally, the authors identified an association between tumor site and survival, however they failed to provide median overall survival and time to recurrence by tumor type. This additional data would provide more insight regarding the patient population and the inherent pathologic characteristics of the CRC cohort. Finally, and most importantly, the authors failed to discuss how recurrences in their cohort were detected. Were recurrences detected by CEA, colonoscopy, or radiographic imaging? This is an important datapoint to include in a study of long-term follow-up because it can provide insight into the optimal long-term surveillance protocol after the initial 5 years of follow-up after resection.

There is increasing evidence that select CRC patients should be followed closely past the initial 5 years of surveillance after curative resection. Unfortunately, guidelines to aid the physician in selecting patients that will benefit from prolonged follow-up are lacking. Large multiinstitutional studies are needed to identify appropriate and applicable surveillance guidelines after the initial 5 years of surveillance following CRC resection.

\section{Acknowledgments}

Funding: None. 


\section{Footnote}

Conflicts of Interest: The authors have no conflicts of interest to declare.

Ethical Statement: The authors are accountable for all aspects of the work in ensuring that questions related to the accuracy or integrity of any part of the work are appropriately investigated and resolved.

Open Access Statement: This is an Open Access article distributed in accordance with the Creative Commons Attribution-NonCommercial-NoDerivs 4.0 International License (CC BY-NC-ND 4.0), which permits the noncommercial replication and distribution of the article with the strict proviso that no changes or edits are made and the original work is properly cited (including links to both the formal publication through the relevant DOI and the license). See: https://creativecommons.org/licenses/by-nc-nd/4.0/.

\section{References}

1. Bray F, Ferlay J, Soerjomataram I, et al. Global cancer statistics 2018: GLOBOCAN estimates of incidence and mortality worldwide for 36 cancers in 185 countries. CA Cancer J Clin 2018;68:394-424.

2. Figueredo A, Rumble RB, Maroun J, et al. Follow-up of patients with curatively resected colorectal cancer: a practice guideline. BMC Cancer 2003;3:26.

3. Deijen CL, Vasmel JE, de Lange-de Klerk ESM, et al. Ten-year outcomes of a randomised trial of laparoscopic versus open surgery for colon cancer. Surgical Endoscopy and Other Interventional Techniques 2017;31:2607-15.

4. Kong L, Peng J, Li J, et al. Prolonged surveillance of colorectal cancer patients after curative surgeries beyond five years of follow-up. Ann Transl Med 2019. doi: 10.21037/atm.2019.10.39.

5. Kohler BA, Ward E, McCarthy BJ, et al. Annual report to

Cite this article as: Caso R, Fabrizio A, Sosin M. Prolonged follow-up of colorectal cancer patients after 5 years: to follow or not to follow, that is the question (and how)! Ann Transl Med 2020;8(5):164. doi: 10.21037/atm.2019.11.40 the nation on the status of cancer, 1975-2007, featuring tumors of the brain and other nervous system. J Natl Cancer Inst 2011;103:714-36.

6. Siegel R, Naishadham D, Jemal A. Cancer statistics, 2013. CA Cancer J Clin 2013;63:11-30.

7. Steele SR, Chang GJ, Hendren S, et al. Practice Guideline for the Surveillance of Patients After Curative Treatment of Colon and Rectal Cancer. Dis Colon Rectum 2015;58:713-25.

8. Frontali A, Benichou B, Valcea I, et al. Is follow-up still mandatory more than 5 years after surgery for colorectal cancer? Updates Surg 2020;72:55-60.

9. Dietel M, Johrens K, Laffert MV, et al. A 2015 update on predictive molecular pathology and its role in targeted cancer therapy: a review focussing on clinical relevance. Cancer Gene Ther 2015;22:417-30.

10. Popat S, Hubner R, Houlston RS. Systematic review of microsatellite instability and colorectal cancer prognosis. J Clin Oncol 2005;23:609-18.

11. Roth AD, Tejpar S, Delorenzi M, et al. Prognostic role of KRAS and BRAF in stage II and III resected colon cancer: results of the translational study on the PETACC-3, EORTC 40993, SAKK 60-00 trial. J Clin Oncol 2010;28:466-74.

12. Marin G, Suarez J, Vera R, et al. Local recurrence after five years is associated with preoperative chemoradiotherapy treatment in patients diagnosed with stage II and III rectal cancer. Int J Surg 2017;44:15-20.

13. National Comprehensive Cancer Network. Rectal Cancer (Version 3), 2019. Available online: https://www.nccn.org/ professionals/physician_gls/pdf/rectal.pdf

14. National Comprehensive Cancer Network. Colon Cancer (Version 3), 2019. Available online: https://www.nccn.org/ professionals/physician_gls/pdf/colon.pdf

15. Kishiki T, Kuchta K, Matsuoka H, et al. The impact of tumor location on the biological and oncological differences of colon cancer: Multi-institutional propensity score-matched study. Am J Surg 2019;217:46-52. 